\title{
Mouse red cell rosette formation and the colchicine sensitivity test: relative usefulness in the differential diagnosis of chronic lymphocytic leukaemia and B lymphocytic lymphoma
}

\author{
PENELOPE J SUGDEN, JS LILLEYMAN \\ From the Department of Haematology, The Children's Hospital, Sheffield S10 2TH
}

SUMMARY Mouse erythrocyte (M) rosette formation and colchicine sensitivity were compared for their ability to differentiate chronic lymphocytic leukaemia (CLL) from B cell non-Hodgkin's lymphoma (NHL) with overspill.

Twenty-two cases of CLL and eight of NHL were studied along with 31 normal adults. Results from the patients in both tests differed significantly from the controls but colchicine sensitivity failed to differentiate them further. $M$ rosettes, on the other hand, while increased in some patients with NHL were, without overlap, much more numerous in those with CLL, and clearly distinguished the two conditions.

A significant autolymphocytotoxic effect of plasma from both study groups was also noted which was not found in the controls.

Where chronic lymphocytic leukaemia (CLL) presents with atypical morphology or a low white count and more malignant B cell non-Hodgkin's lymphomas (NHL) display blood and bone marrow dissemination, distinguishing these conditions morphologically and immunologically can be difficult. Since the prognosis and treatment are not the same a simple discriminatory test is potentially useful.

Selective ultrasensitivity of CLL lymphocytes to killing by low concentrations of colchicine was shown in 1972 by Thomson et al, ${ }^{1}$ and this characteristic is thought to be a sensitive method not only of detecting such abnormal cells but also of assessing the size of the population. ${ }^{2}$ Alternatively, more than $95 \%$ of cases of CLL have been reported to have a large proportion of peripheral lymphocytes with a specific ability to form rosettes with mouse erythrocytes; this proportion varying between $40 \%$ and $90 \% .^{3} 4$

To assess the ability of these two tests to distinguish CLL lymphocytes from those of other B lymphoproliferative disorders, a study was undertaken comparing the two techniques in a selected group of patients where conventional criteria allowed a confident diagnosis of CLL or NHL.
Material and methods

PATIENTS STUDIED

Adult patients encountered over two years were examined. Diagnosis of CLL was made on the basis of clinical findings and an increase in non- $T$ lymphocytes staining either weakly or not at all for surface immunoglobulin. B cell NHL was diagnosed on the basis, in addition to the clinical picture, of increased abnormal lymphocytes in the blood which stained strongly for surface immunoglobulin. In all cases where the lymphocytes were tested with specific light chain typing sera their monoclonal nature was established.

Eight other patients with non-T lymphoproliferative disease encountered during the study period were excluded from analysis as their findings prevented confident classification.

Normal controls were adult members of hospital staff or outpatients with non-haematological, noninfective complaints.

\section{TECHNIQUES}

Lymphocytes were separated from $5 \mathrm{ml}$ heparinised blood by centrifugation on a Ficoll-Triosil mixture, washed twice and resuspended in medium 199 at a concentration of $0.25 \times 10^{6} / 0.4 \mathrm{ml}$. T lymphocytes 376 
were identified by sheep-E-rosette formation.

Surface immunoglobulin was detected by direct immunofluorescence of $1 \times 10^{6}$ lymphocytes which had been incubated at $37^{\circ} \mathrm{C}$ in medium 199 for at least $30 \mathrm{~min}$ and washed to remove extrinsic bound immunoglobulin.

\section{MOUSE (M) ROSETTE FORMATION}

Mouse red cells were obtained from CBA or C57B1 mice, which have both been shown to give the same results. ${ }^{3}$ They were washed three times and made up to $1 \% \mathrm{vol} / \mathrm{vol}$ in medium $199 / 20 \%$ fetal calf serum, and were shown to give the same results after storing for up to 2 weeks at $4^{\circ} \mathrm{C}$. Lymphocytes were tested in duplicate by mixing $0.25 \times 10^{6}$ with $0.25 \mathrm{ml}$ mouse red cells, supplementing with $20 \%$ fetal calf serum, centrifuging gently $(200 \mathrm{~g})$ for $5 \mathrm{~min}$ at room temperature and then incubating at room temperature for $1 \mathrm{~h}$. After gentle resuspension and staining with $0.3 \%$ methylene blue the cells were examined in a haemocytometer and the percentage of 200 lymphocytes with more than two mouse red cells adhering was counted.

\section{COLCHICINE SENSITIVITY TEST}

Lymphocytes were tested in duplicate for sensitivity to colchicine at three molar concentrations $\left(10^{-7} M\right.$, $10^{-6} \mathrm{M}$ and $10^{-5} \mathrm{M}$ ) compared with a background without colchicine. Lymphocytes $\left(0.25 \times 10^{6}\right)$ in medium 199 supplemented to $30 \%$ with autologous plasma or fetal calf serum were incubated with and without colchicine at $37^{\circ} \mathrm{C}$ for $24 \mathrm{~h}$. The proportion of pyknotic cells was determined on a cytocentrifuge preparation made from each culture, wet-fixed in Susa's fixative for $10 \mathrm{~min}$, washed in running tap water for $15 \mathrm{~min}$, stained for $2 \mathrm{~min}$ in Mayer's haemalum and washed for a further $15 \mathrm{~min}$. The slides were dried quickly with a fan and examined "blind," with the label obscured, under a light microscope. Five hundred cells were examined per slide by the same observer, and each was counted as pyknotic or not. Pyknotic cells were shrunken, rounded and contained densely staining nuclear material. The mean percentage of pyknotic cells in cultures without colchicine was subtracted from the mean percentage pyknotic at each dose.

\section{STATISTICAL METHODS}

Student's $t$ test was used to determine the significance of the difference between mean values from patients and controls. The paired $t$ test was used to compare cell death in autologous plasma and fetal calf serum. Correlations were done using Pearson's product moment correlation coefficient.

\section{Results}

Twenty-two patients with CLL, eight with NHL and 31 controls were studied. The lymphocyte counts ranged from $285 \times 10^{9} / 1$ to $6.0 \times 10^{9} / 1$ in the CLL patients and from $74 \times 10^{9} / 1$ to $6.0 \times 10^{9} / 1$ in those with NHL. The mean, range and standard error of the proportion of mouse rosetting and colchicine sensitive lymphocytes in the groups studied are shown in the Table.

It was found at an early stage that the percentage of cells dying in CLL or NHL plasma without the addition of colchicine was often very high $(37-95 \%)$, much higher than the control backgrounds. Incubating cells from nine of these patients (six CLL, three NHL) in both autologous plasma and fetal calf serum led to the discovery that in the latter, in all cases, the backgrounds were much lower (11-54\%) mostly within the normal range $(5-24 \%)$. The difference between the results in the two types of supplement was highly significant $(p<0.001)$ indicating that patient's plasma had a lymphotoxic effect. Following this discovery all patient colchicine sensitivity cultures were incubated with fetal calf serum rather than autologous plasma as supplement. This cytotoxic effect was not seen in the control subjects.

Chronic lymphocytic leukaemia lymphocytes were ultrasensitive to colchicine at all three doses compared to the controls ( $p<0.001)$, as were those from the NHL patients $(p<0.001)$. The two groups of patients did not differ significantly from each other, however $(\mathrm{p}>0 \cdot 1)$.

$M$ rosette formation results did, on the other hand, show a difference (see Table). While both the mean CLL value and the mean NHL values were significantly higher than the control value $(p<0.001$, $<0.002$ respectively), the NHL value was clearly significantly lower than the CLL value $(p<0.001)$. In addition, whereas the NHL values overlapped with control values, those of the CLL patients did

Mouse E rosetting and colchicine sensitivity test results

\begin{tabular}{lcccc}
\hline & $\begin{array}{c}\text { \% mouse } \\
\text { E rosettes }\end{array}$ & \multicolumn{2}{l}{$\%$ pyknotic cells with colchicine } \\
\cline { 3 - 5 } & & $10^{-7} M$ & $10^{-6} M$ & $10^{-5} M$ \\
\hline CLL & & & & \\
Mean & $56 \cdot 1$ & $41 \cdot 5$ & $40 \cdot 9$ & $40 \cdot 7$ \\
Patients SEM & $3 \cdot 3$ & $7 \cdot 2$ & $6 \cdot 8$ & $6 \cdot 4$ \\
Range & $29-80$ & $5-77$ & $19-79$ & $9-75$ \\
NHL & & & & \\
Mean & $5 \cdot 5$ & $27 \cdot 8$ & $35 \cdot 8$ & $32 \cdot 0$ \\
Patients SEM & $1 \cdot 9$ & $6 \cdot 3$ & $5 \cdot 7$ & $7 \cdot 0$ \\
Range & $0-15$ & $18-52$ & $22-50$ & $16-58$ \\
Controls & & & & \\
Mean & $1 \cdot 6$ & $5 \cdot 4$ & $5 \cdot 7$ & $5 \cdot 7$ \\
SEM & $0 \cdot 3$ & $1 \cdot 2$ & $2 \cdot 2$ & $1 \cdot 7$ \\
Range & $0-8 \cdot 5$ & $0-12$ & $0-22$ & $0-18$ \\
& & & & \\
\hline
\end{tabular}


not, nor did they overlap with the NHL values (Figure).

Finally the numbers of colchicine-sensitive and $M$ rosetting cells in each patient were compared to each other and to the absolute lymphocyte counts (as logarithms). Although there was a tendency for the proportion of abnormal cells measured by either technique to correlate with the number of circulating lymphocytes (apart from $M$ rosettes in NHL) this did not achieve significance $(p<0.1,>0.05)$. Similarly, there was no correlation between abnormal cell numbers estimated by the two techniques.

\section{Discussion}

It is clear from this study that $\mathrm{M}$ rosette formation can be used with reasonable confidence to distinguish CLL lymphocytes from the neoplastic cells of B cell NHL. The colchicine sensitivity test, on the other hand, cannot.

As the main object was to define a useful discriminatory test in an occasionally confusing clinical situation it would be unwise to draw any further conclusion from these findings on the specificity of either technique, or what particular cell (if any) each identifies.

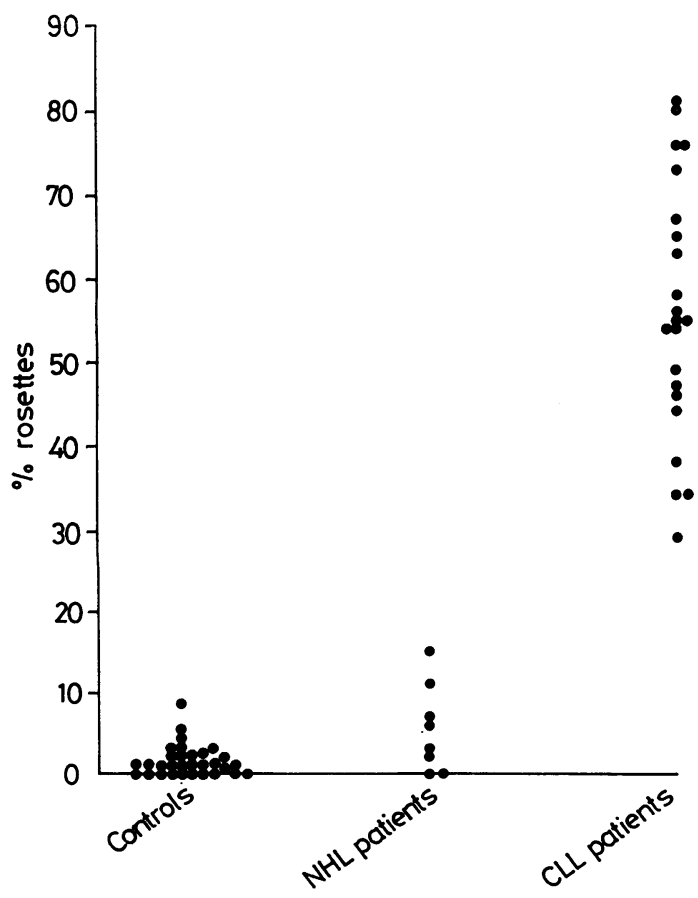

Mouse red cell rosette numbers in patients with $C L L$ and NHL and normal controls.
Colchicine sensitive cells in lymphomas other than CLL have been noted before ${ }^{2} 5$ and are thought to represent a fairly wide variety of immature, or abnormal, B cells. ${ }^{5}$ Normal B cells, however, are not apparently colchicine-sensitive, either those circulating ${ }^{5}$ or those in lymph nodes. ${ }^{6}$ Our own results would confirm that peripheral mixed normal lymphocytes are also resistant, and so the test may be helpful in distinguishing neoplastic from reactive lymphoproliferation.

$M$ rosette formation, in contrast, appears to be a fairly specific feature of the CLL lymphocyte and has rarely been observed in other conditions. Exceptions include plasmacytomata, Waldenström's macroglobulinaemia, ${ }^{7}$ and a proportion of patients with hairy cell leukaemia ${ }^{8}$ As none of these disorders is likely to be confused with CLL if appropriate protein studies and cytochemistry are performed, they do not diminish the usefulness of the test. Ability to form $\mathbf{M}$ rosettes probably marks a fairly specific stage of $B$ cell differentiation to which the CLL lymphocyte corresponds.

The lack of correlation between colchicinesensitive cells and $\mathbf{M}$ rosettes formed by our patients and their lymphocyte counts is not surprising as a similar lack of correlation has been previously noted $^{\mathbf{3} 9}$ and presumably simply reflects a variation in the number of normal lymphocytes present.

The incidental discovery of a cytotoxic effect of patients' plasma on their own cells is of more interest. Other workers have noted that CLL lymphocytes survive less well in cultures without colchicine than normal lymphocytes, ${ }^{59}$ and a nonantibody lymphotoxic factor in serum from a patient with CLL has been demonstrated using a cytotoxicity test. ${ }^{10}$ CLL serum has also been found to inhibit lymphocyte protein and nucleic acid synthesis induced by PHA, ${ }^{11} 12$ but the nature and specificity of such inhibitory and cytotoxic activity, however, remains obscure at present.

We acknowledge the help of $\mathrm{Dr} R$ Walker, who provided the mouse red blood cells, and that of the physicians at the Royal Hallamshire Hospital whose patients we studied.

JSL is in receipt of a grant from the Leukaemia Research Fund.

References

1 Thomson AER, O'Connor TWE, Wetherley-Mein G. Killing and characterising action of colchicine in vitro on lymphocytes of chronic lymphocytic leukaemia. Scand J Haematol 1972;9:231-47.

${ }^{2}$ Schrek R. Sensitivity to colchicine as a test for leukaemic lymphocytes. $N$ Engl J Med 1975;293:151.

${ }^{8}$ Stathopoulos G, Elliott EV. Formation of mouse or sheep 
red-blood-cell rosettes by lymphocytes from normal and leukaemic individuals. Lancet $1974 ; \mathrm{i}: 600-1$.

${ }^{4}$ Dobozy A, Huniyadi J, Krizsa F, Kenderessy AS, Simon N. A comparative study of the shedding process of mouse erythrocyte binding receptors on peripheral blood lymphocytes from healthy donors and patients suffering from chronic lymphocytic leukaemia. Exp Hematol 1980;8:690-3.

${ }^{5}$ Scarffe JH, Prudhoe J, Garrett JV, Crowther D. Colchicine ultrasensitivity of peripheral blood lymphocytes from patients with non-Hodgkin's lymphoma. Br J Cancer $1980 ; 41: 593-601$.

${ }^{6}$ Schrek R, Molnar Z, Stefani SS. Cytology and colchicine sensitivity of viable cells from lymph nodes with malignant lymphoma. Cancer 1978;41:1845-56.

7 Gupta S, Good RA, Siegal FP. Rosette formation with mouse erythrocytes: III: Studies in patients with primary immunodeficiency and lymphoproliferative disorders. Clin Exp Immunol 1976;26:204-13.

${ }^{8}$ Burns GF, Cawley JC. Spontaneous mouse erythrocyte rosette formation: Correlation with surface immunoglobulin phenotype in hairy-cell leukaemia. Clin Exp Immunol 1980;39:83-9.

Thomson AER, O'Connor TWE. Selective killing by colchicine in vitro of lymphocytes in chronic lymphocytic leukaemia. Proceedings of the 8th Leucocyte Culture Conference 1974;665-71.

${ }^{10}$ Dickson A. Lymphocytotoxins in leukaemia. $\mathrm{Br} \mathrm{Med} \mathrm{J}$ 1972;ii:209-11.

1 Spenglar GA, Stjernholm RL. A transient lymphotoxic serum factor in a patient with chronic lymphocytic leukaemia. Am J Med Sci 1972;263:241-52.

12 Sugden PJ. Autoinhibition of PHA-response by chronic lymphocytic leukaemia serum. Lancet 1974;ii:596-7.

Requests for reprints to: Dr JS Lilleyman, Department of Haematology, The Children's Hospital, Sheffield S10 2TH, England. 